\title{
SISTEMA RTP: UMA TÉCNICA PODEROSA PARA O MONITORAMENTO DA FORMAÇÃO DE NANOTUBOS DE CARBONO DURANTE O PROCESSO POR DEPOSIÇÃO DE VAPOR QUÍMICO
}

\author{
Juliana Cristina Tristão, Flávia Cristina Camilo Moura e Rochel Montero Lago* \\ Departamento de Química, Instituto de Ciências Exatas, Universidade Federal de Minas Gerais, 31270-901 Belo Horizonte - MG, Brasil \\ Karim Sapag \\ Laboratorio de Ciencias de Superficies y Medios Porosos, Universidade Nacional de San Luis, 5700, San Luis, Argentina
}

Recebido em 1/4/09; aceito em 18/2/10; publicado na web em 3/5/10

\begin{abstract}
TPR SYSTEM: A POWERFUL TECHNIQUE TO MONITOR CARBON NANOTUBE FORMATION DURING CHEMICAL VAPOUR DEPOSITION. In this work, a TPR (Temperature Programmed Reduction) system is used as a powerful tool to monitor carbon nanotubes production during CVD (Chemical Vapour Deposition), The experiments were carried out using catalyst precursors based on $\mathrm{Fe}$-Mo supported on $\mathrm{Al}_{2} \mathrm{O}_{3}$ and methane as carbon source. As methane reacts on the $\mathrm{Fe}$ metal surface, carbon is deposited and $\mathrm{H}_{2}$ is produced. TPR is very sensitive to the presence of $\mathrm{H}_{2}$ and affords information on the temperature where catalyst is active to form different forms of carbon, the reaction kinetics, the catalyst deactivation and carbon yields.
\end{abstract}

Keywords: carbon nanotubes; CVD; TPR.

\section{INTRODUÇÃO}

Nos últimos anos, os nanotubos de carbono (NTC) vêm despertando grande interesse de pesquisadores no mundo por apresentarem extraordinárias propriedades mecânicas, elétricas e térmicas. Estes materiais podem ser produzidos por diferentes métodos, sendo o mais investigado o processo por Deposição de Vapor Químico (CVD) baseado na decomposição térmica de fontes de carbono gasosas, como $\mathrm{CH}_{4}, \mathrm{C}_{2} \mathrm{H}_{4}$ ou outras moléculas orgânicas, na superfície de catalisadores metálicos nanoparticulados. ${ }^{1,2}$ Apesar de um grande número de catalisadores e fontes de carbono ter sido investigado para a síntese de NTC por CVD, informação sobre a cinética de reação é ainda escassa. Visto que o CVD é considerado o processo mais promissor para a produção industrial de NTC, informações cinéticas são especialmente relevantes para a definição do processo e dos projetos dos reatores industriais.

Alguns poucos trabalhos têm explorado diferentes técnicas para o estudo da cinética de formação de NTC. Citamos, por exemplo, o uso de termogravimetria no qual uma microbalança foi conectada na zona de reação do sistema CVD e a massa de carbono foi monitorada ao longo do tempo..$^{3-5}$ Nestes trabalhos foi possível obter informações tais como a razão de deposição de carbono, o rendimento e a expressão cinética da reação. Outras estratégias para estudo da formação de NTC são a medida de corrente elétrica entre eletrodos para controlar a quantidade de NTC formados, ${ }^{6}$ a aplicação de uma sonda TEOM (Tapered Element Oscillating Microbalance) ${ }^{7}$ análise dos produtos gasosos da reação através de um sistema $\mathrm{GC} / \mathrm{MS}^{8}$ e, o uso da técnica de interferência ótica. ${ }^{9-11}$

Neste trabalho, é proposto o uso da técnica de redução à temperatura programada (RTP) para o monitoramento do crescimento de nanotubos de carbono por CVD. Um experimento típico de RTP monitora o consumo de $\mathrm{H}_{2}$ durante a redução de uma amostra sob fluxo de gás redutor (usualmente $\left.\mathrm{H}_{2}\right)$ diluído em gás inerte $\left(\mathrm{N}_{2}\right.$ ou $\mathrm{Ar}$ ), enquanto a temperatura é aumentada linearmente com o tempo. A RTP é considerada uma ferramenta poderosa na análise da cinética de redução de precursores de catalisadores na forma oxidada e revela informações relacionadas com as propriedades redox do material, sobre a temperatura de redução, estado de valência de óxidos metálicos, interação entre óxidos metálicos e o suporte, indicação da formação

*e-mail: rochel@ufmg.br de ligas em catalisadores bimetálicos, mecanismos e cinéticas de redução, entre outros. ${ }^{12-14}$

O objetivo deste trabalho foi o uso de um sistema RTP para monitorar a formação de estruturas de carbono durante a reação CVD usando diferentes precursores de catalisador. Este monitoramento é possível, devido à formação de $\mathrm{H}_{2}$ durante a produção dos NTC, como ilustrado para metano na Equação 1:

$$
\mathrm{CH}_{4}+\text { catalisador } \rightarrow \mathrm{C}_{\mathrm{NTC}}+2 \mathrm{H}_{2}
$$

A representação esquemática do sistema RTP pode ser vista na Figura 1.

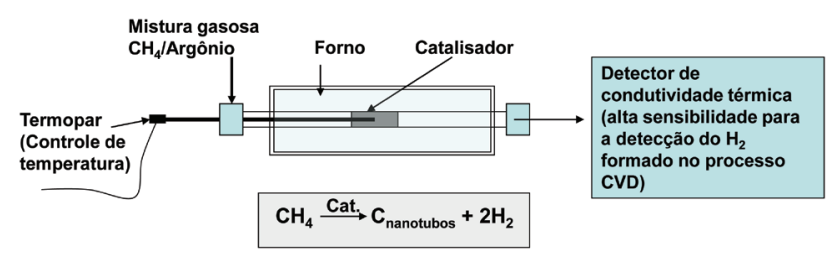

Figura 1. Representação esquemática simplificada do sistema RTP para monitorar a formação de NTC a partir da reação de $\mathrm{CH}_{4}$ em catalisadores

A técnica RTP permite o monitoramento da formação de $\mathrm{H}_{2}$ com alta sensibilidade, o estudo do efeito da temperatura de reação, estudo da cinética de reação em condições isotérmicas e estudo da desativação dos catalisadores.

Neste trabalho foram utilizados dois tipos de catalisadores, e.g., Fe-Mo e perovskitas do tipo $\mathrm{LaFe}_{\mathrm{x}} \mathrm{Mn}_{\mathrm{y}} \mathrm{Mo}_{\mathrm{z}} \mathrm{O}_{3}$, ambos suportados em $\mathrm{Al}_{2} \mathrm{O}_{3}$ nanoparticulada. Estes catalisadores mostraram bons resultados para a produção de NTC de parede simples em trabalhos anteriores ${ }^{15-17}$ e foram utilizados aqui para ilustrar o potencial da técnica.

\section{PARTE EXPERIMENTAL}

Foram preparadas as seguintes perovskitas: $\mathrm{LaFe}_{0,90} \mathrm{Mn}_{0,10} \mathrm{O}_{3}$ e $\mathrm{LaFe}_{0,90} \mathrm{Mn}_{0,08} \mathrm{Mo}_{0,02} \mathrm{O}_{3}$. No preparo das perovskitas, 0,5 mol de ácido cítrico anidro - AC - foram dissolvidos em $2 \mathrm{~mol}$ de água a $60{ }^{\circ} \mathrm{C}$, seguido da adição de $1 \mathrm{~mol}$ de $\mathrm{La}\left(\mathrm{NO}_{3}\right)_{3} \cdot 6 \mathrm{H}_{2} \mathrm{O}$ e diferentes 
proporções dos sais metálicos $\mathrm{Fe}\left(\mathrm{NO}_{3}\right)_{3} \cdot 9 \mathrm{H}_{2} \mathrm{O}, \mathrm{Mn}\left(\mathrm{NO}_{3}\right)_{2} \cdot 4 \mathrm{H}_{2} \mathrm{O}$ e $\mathrm{Mo}(\mathrm{acac})_{2} \mathrm{O}_{2}$. Depois da completa dissolução dos sais a $60^{\circ} \mathrm{C}$ por 2 $\mathrm{h}$, foram adicionados à solução $400 \mathrm{mmol}$ de etileno glicol - EG - e $\mathrm{Al}_{2} \mathrm{O}_{3}$ nanoparticulada (Aldrich) nas proporções de 25, 33 e 50\% de perovskita, sendo então a temperatura lentamente elevada a 90 ${ }^{\circ} \mathrm{C}$. Esta etapa remove o excesso de água e proporciona a reação de poliesterificação entre AC e EG. A mistura assim obtida foi aquecida sob agitação constante a aproximadamente $90^{\circ} \mathrm{C}$ por $7 \mathrm{~h}$, produzindo uma massa alaranjada mais viscosa. A resina obtida foi colocada em uma manta aquecedora a $400-450{ }^{\circ} \mathrm{C}$ por $2 \mathrm{~h}$ e calcinada em mufla a $800{ }^{\circ} \mathrm{C} \mathrm{em}$ ar por $6 \mathrm{~h}$.

$\mathrm{O}$ catalisador clássico $\mathrm{Fe}-\mathrm{Mo} / \mathrm{Al}_{2} \mathrm{O}_{3}$ foi preparado na proporção Fe:Mo: $\mathrm{Al}_{2} \mathrm{O}_{3}$ de 1:0,16:8 por impregnação da $\mathrm{Al}_{2} \mathrm{O}_{3}$ nanoparticulada pelos sais de ferro e molibdênio.

As imagens obtidas por microscopia eletrônica de varredura (MEV) foram feitas no equipamento Jeol JSM $840 \mathrm{~A}$. Os produtos de carbono foram caracterizados por espectroscopia Raman (Renishaw) com comprimento de onda de excitação de $633 \mathrm{~nm}$. Os espectros obtidos representam a média de 5 a 10 diferentes pontos para minimizar a dispersão da posição da amostra.

O monitoramento da formação de nanotubos de carbono foi realizado por RTP em um equipamento Chem BET 3000 TPR Quantachrome. Neste estudo, $30 \mathrm{mg}$ do precursor catalítico foram inicialmente submetidos a um fluxo de $20 \mathrm{~mL} \mathrm{~min}^{-1}$ de Ar contendo $5 \% \mathrm{H}_{2}$ e aquecidos a uma taxa de $10{ }^{\circ} \mathrm{C} \min ^{-1}$ até $900{ }^{\circ} \mathrm{C}$ e, então, mantidos nesta temperatura por $1 \mathrm{~h}$. Em seguida, o fluxo de $\mathrm{H}_{2}$ foi fechado e a temperatura foi reduzida até $400{ }^{\circ} \mathrm{C}$, sob fluxo de argônio. A $400{ }^{\circ} \mathrm{C}$, foi injetado um fluxo de $\mathrm{Ar}$ de $20 \mathrm{~mL} \mathrm{~min}^{-1}$ contendo $5 \%$ $\mathrm{CH}_{4}$ e, após estabilização do sistema, a temperatura foi novamente elevada até $900^{\circ} \mathrm{C} \mathrm{a} 10^{\circ} \mathrm{C} \mathrm{min}^{-1}$ permanecendo a $900^{\circ} \mathrm{C}$ por $1 \mathrm{~h}$ para formação dos nanotubos de carbono. A calibração do equipamento foi realizada através da injeção de $\mathrm{H}_{2}$ ultrapuro (1 a $200 \mu \mathrm{L}$ ) através de microsseringas para gases. Todos os rendimentos foram calculados em função da quantidade de ferro presente no catalisador. Os rendimentos obtidos por TG consideraram a perda de massa devido à oxidação do carbono pelo ar.

\section{RESULTADOS E DISCUSSÃO}

\section{Catalisadores selecionados}

Os estudos de formação de NTC por RTP foram realizados utilizando-se dois tipos de catalisadores já investigados em nosso grupo de pesquisa, i.e., Fe-Mo suportado em $\mathrm{Al}_{2} \mathrm{O}_{3}$ e perovskitas do tipo $\mathrm{LaFe}_{\mathrm{x}} \mathrm{Mo}_{\mathrm{y}} \mathrm{Mn}_{\mathrm{z}} \mathrm{O}_{3}$ suportadas em $\mathrm{Al}_{2} \mathrm{O}_{3}$. O catalisador $\mathrm{Fe}-\mathrm{Mo} / \mathrm{Al}_{2} \mathrm{O}_{3}$, bastante investigado na literatura, ${ }^{18-20}$ pode ser preparado por simples impregnação e mostra alta eficiência na produção de NTC de parede simples. As perovskitas funcionam como precursor do catalisador e são pré-reduzidas com $\mathrm{H}_{2}$ para formar partículas de $\mathrm{Fe}^{0}$ dispersas no suporte $\mathrm{Al}_{2} \mathrm{O}_{3}$. A Equação 2 mostra este processo de forma simplificada:

$\mathrm{LaFe}_{\mathrm{x}} \mathrm{Mo}_{\mathrm{y}} \mathrm{Mn}_{\mathrm{z}} \mathrm{O}_{3} / \mathrm{Al}_{2} \mathrm{O}_{3}+\mathrm{H}_{2} \rightarrow \mathrm{Fe}-\mathrm{Mo} /\left(\mathrm{La}_{2} \mathrm{O}_{3}, \mathrm{MnO}, \mathrm{Al}_{2} \mathrm{O}_{3}\right)$

Um estudo detalhado destes catalisadores mostrou que o teor da perovskita na $\mathrm{Al}_{2} \mathrm{O}_{3}$ afeta profundamente a distribuição de tamanho das nanopartículas de $\mathrm{Fe}^{0} \mathrm{e}$, portanto, as diferentes formas de carbono produzidas. ${ }^{15,16}$

\section{Monitoramento da formação de nanotubos de carbono com RTP}

Os experimentos de síntese de NTC foram realizados dentro do forno do equipamento RTP, simulando-se o processo CVD de acordo com as seguintes etapas: $1^{\mathrm{a}}$ : redução do catalisador com $\mathrm{H}_{2}(5 \%) / \mathrm{Ar}$ até $900{ }^{\circ} \mathrm{C} / 1 \mathrm{~h}$, para formação da fase Fe-Mo ativa;

$2^{\mathrm{a}}$ : resfriamento do reator para $400{ }^{\circ} \mathrm{C}$ e troca do gás $\mathrm{H}_{2}(5 \%) /$ Ar para $\mathrm{CH}_{4}(5 \%) / \mathrm{Ar}$

$3^{\mathrm{a}}$ : aquecimento do reator a $10{ }^{\circ} \mathrm{C} \mathrm{min}^{-1}$ até $900{ }^{\circ} \mathrm{C} / 1 \mathrm{~h}$ com monitoramento da formação de $\mathrm{H}_{2}$ no processo.

A Figura 2 ilustra estes processos obtidos para o catalisador clássico $\mathrm{Fe}-\mathrm{Mo} / \mathrm{Al}_{2} \mathrm{O}_{3}$.

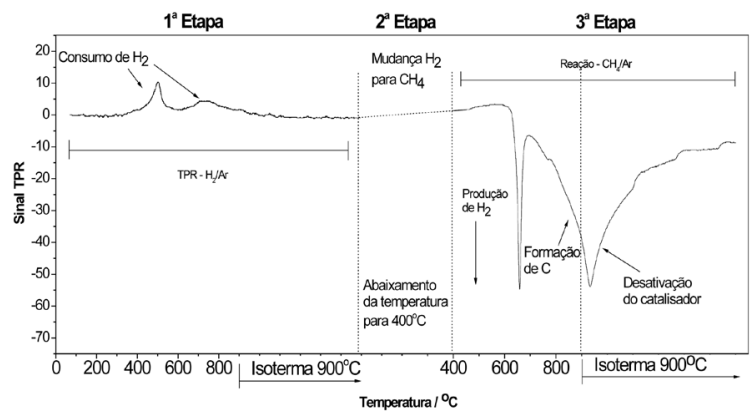

Figura 2. Redução à temperatura programada (RTP) do catalisador clássico $\mathrm{Fe}-\mathrm{Mo} / \mathrm{Al}_{2} \mathrm{O}_{3}\left(1^{a}\right.$ etapa $)$ e acompanhamento da formação de $\mathrm{H}_{2}$ no processo CVD ( $3^{a}$ etapa $)$

A $1^{\text {a }}$ etapa da Figura 2 mostra dois picos principais de redução do catalisador, o primeiro em $500{ }^{\circ} \mathrm{C}$ - que basicamente corresponde à redução da fase $\mathrm{Fe}_{2} \mathrm{O}_{3}$ para $\mathrm{Fe}_{3} \mathrm{O}_{4}$ e o segundo pico em $730^{\circ} \mathrm{C}$ relacionado à formação da fase $\mathrm{FeO}$, a qual é reduzida em seguida para $\mathrm{Fe}^{0} .^{21}$

Após a $1^{\mathrm{a}}$ etapa de redução do catalisador $\mathrm{Fe}-\mathrm{Mo} / \mathrm{Al}_{2} \mathrm{O}_{3}$, utilizouse a mistura gasosa $\mathrm{CH}_{4} / \mathrm{Ar}$ e realizou-se o processo CVD até 900 ${ }^{\circ} \mathrm{C}$. Análises da amostra por MEV e por Raman (Figura 3) sugerem a formação de NTC de paredes simples com diâmetros estimados de aproximadamente $0,90-1,71 \mathrm{~nm}$, calculados a partir dos modos RBM do espectro Raman. ${ }^{22} \mathrm{O}$ espectro Raman, Figura 3, revela também a presença de uma banda entre 1500 e $1600 \mathrm{~cm}^{-1}$ referente aos modos tangenciais do plano grafítico (banda TM), característica de material grafitizado e da banda $\mathrm{D}$, em aproximadamente $1325 \mathrm{~cm}^{-1}$, relacionada à presença de defeitos nos NTC ou de material desordenado, i.e., carbono amorfo. A alta intensidade do pico da banda TM no espectro indica um bom arranjo da rede hexagonal de grafite.

No processo CVD ( $3^{\mathrm{a}}$ etapa), observa-se um pico agudo negativo em aproximadamente $660^{\circ} \mathrm{C}$ que está relacionado à formação rápida de $\mathrm{H}_{2}$ seguida também de uma rápida diminuição. Esta formação de $\mathrm{H}_{2}$ a temperaturas relativamente baixas sugere a presença de uma fase catalítica muito ativa que reage com metano formando $\mathrm{C}$ e $\mathrm{H}_{2} \mathrm{e}$ se desativa facilmente (Equação 3).

$$
\mathrm{Fe}_{\text {ativo }}+\mathrm{CH}_{4} \rightarrow \mathrm{Fe} / \mathrm{C} \text { (desativado) }+2 \mathrm{H}_{2}
$$

Esta fase ativa pode estar relacionada com a presença de partículas de $\mathrm{Fe}^{0}$ muito pequenas e altamente dispersas. Análises Raman após CVD interrompido a $700{ }^{\circ} \mathrm{C}$ mostraram somente a presença de uma banda $\mathrm{D}$ pouco intensa em $1340 \mathrm{~cm}^{-1}$. Estes resultados sugerem que o processo CVD na faixa de temperatura de $600-700{ }^{\circ} \mathrm{C}$ leva à formação essencialmente de carbono amorfo.

Após este pico agudo, observa-se uma diminuição gradativa do sinal RTP entre 700 e $900{ }^{\circ} \mathrm{C}$, o que indica a formação lenta de $\mathrm{H}_{2}$ nesta faixa de temperatura. $\mathrm{Na}$ fase isotérmica a $900{ }^{\circ} \mathrm{C}$, continua sendo produzido $\mathrm{H}_{2}$ por alguns minutos. No entanto, observa-se em seguida o aumento do sinal RTP referente à diminuição da produção de $\mathrm{H}_{2}$. Esta variação no sinal RTP deve estar relacionada com a etapa de formação catalítica de NTC e outras estruturas grafíticas (Equação 4) seguido da desativação do catalisador (Equação 3). 


$$
\mathrm{CH}_{4}+\text { Catalisador } \rightarrow \mathrm{C}_{\mathrm{NTC} \text { e est. grafíticas }}+2 \mathrm{H}_{2}
$$

O processo CVD foi também conduzido para os catalisadores baseados nas perovskitas $\mathrm{LaFe}_{0,90} \mathrm{Mn}_{0,10} \mathrm{O}_{3}$ (pura), $\mathrm{LaFe}_{0,90} \mathrm{Mn}_{0,08} \mathrm{Mo}_{0,02} \mathrm{O}_{3}$ (pura) e $\mathrm{LaFe}_{0,90} \mathrm{Mn}_{0,08} \mathrm{Mo}_{0,02} \mathrm{O}_{3}$ com teores de 25, 33 e 50\% suportadas em $\mathrm{Al}_{2} \mathrm{O}_{3}$. Os catalisadores foram também pré-reduzidos de acordo com a $1^{\mathrm{a}}$ etapa da Figura 2. Após $\mathrm{CVD}$ a $900^{\circ} \mathrm{C}$ todos os catalisadores foram analisados por espectroscopia Raman e por MEV.

As imagens MEV (Figura 1S, material suplementar) mostraram para a perovskita pura $\mathrm{LaFe}_{0,90} \mathrm{Mn}_{0,08} \mathrm{Mo}_{0,02} \mathrm{O}_{3}$ a formação de grande quantidade de filamentos de carbono com diâmetros variando de 30 a $200 \mathrm{~nm}$. Por outro lado, quando a perovskita é suportada em $\mathrm{Al}_{2} \mathrm{O}_{3}$, observa-se claramente a formação de filamentos de carbono com valores de diâmetros menores, especialmente para o compósito com $25 \%$ de perovskita.

As análises Raman (Figura 4) mostraram a presença da banda TM em ca. $1580-1600 \mathrm{~cm}^{-1}$ característica de materiais grafíticos. A presença de um ombro em ca. $1620 \mathrm{~cm}^{-1}$ que aparece para as perovskitas $\mathrm{P}$ (pura) e $\mathrm{P}(50 \%) / \mathrm{Al}_{2} \mathrm{O}_{3}$ sugere a formação de nanotubos de paredes múltiplas (NTPM). Para todas as perovskitas observou-se a ocorrência do pico $\mathrm{D}$ em $1330 \mathrm{~cm}^{-1}$ referente à presença de defeitos e carbono amorfo. Para a perovskita $\mathrm{LaFe}_{0,90} \mathrm{Mn}_{0,08} \mathrm{Mo}_{0,02} \mathrm{O}(\mathrm{P})$ não foram observados picos na região de baixo número de onda, onde aparecem os modos de respiração radial (RBM), indicando que nanotubos de paredes simples (NTPS) não foram formados. Entretanto, a amostra $\mathrm{P}(50 \%) / \mathrm{Al}_{2} \mathrm{O}_{3}$ apresentou picos $\mathrm{RBM}$ com baixa intensidade entre 130 e $200 \mathrm{~cm}^{-1}$, o que indica a formação de NTPS. Quando a concentração da perovskita sobre a alumina diminui para 33 e $25 \%$, vários aspectos no espectro Raman mudam, e.g, a intensidade relativa dos modos RBM aumenta e a posição da banda TM

a)

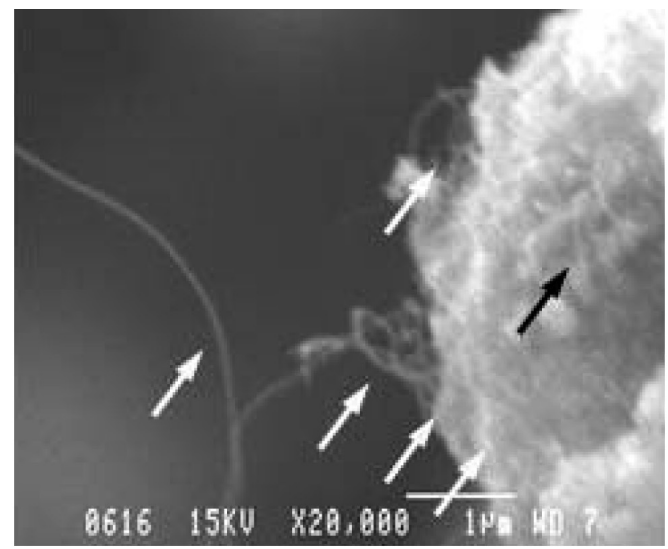

b)

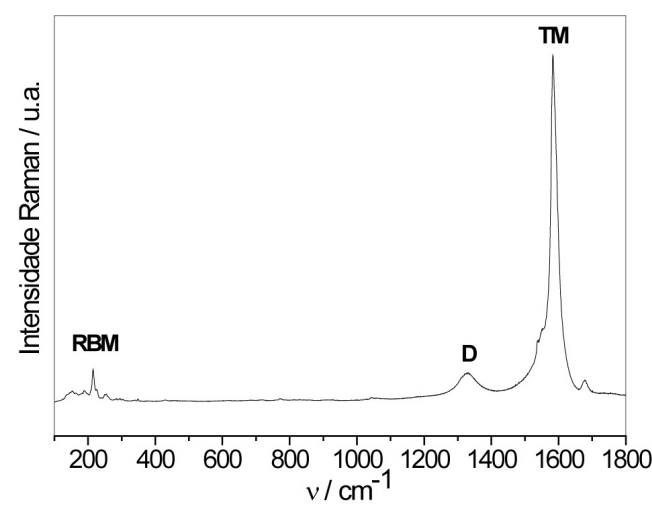

Figura 3. Análises $\mathrm{MEV}$ (a) e Raman (b) da amostra $\mathrm{Fe}-\mathrm{Mo} / \mathrm{Al}_{2} \mathrm{O}_{3}$ após CVD a $900^{\circ} \mathrm{C}$ é deslocada de 1583 para $1592 \mathrm{~cm}^{-1}$ sugerindo a formação de mais NTPS, ${ }^{4}$ a intensidade da banda D diminui, sugerindo a formação de menos defeitos ou de menor quantidade de carbono amorfo e, não são observados ombros em ca. $1620 \mathrm{~cm}^{-1}$ indicando a ausência de NTPM. O efeito do Mo na formação dos NTC foi também investigado, comparando-se os resultados obtidos para as perovskitas $\mathrm{LaFe}_{0,90} \mathrm{Mn}_{0,10} \mathrm{O}_{3} \mathrm{e} \mathrm{LaFe}{ }_{0,90} \mathrm{Mn}_{0,08} \mathrm{Mo}_{0,02} \mathrm{O}_{3}$. Verificou-se que a presença do Mo proporciona a formação de materiais mais bem grafitizados. ${ }^{15,16}$

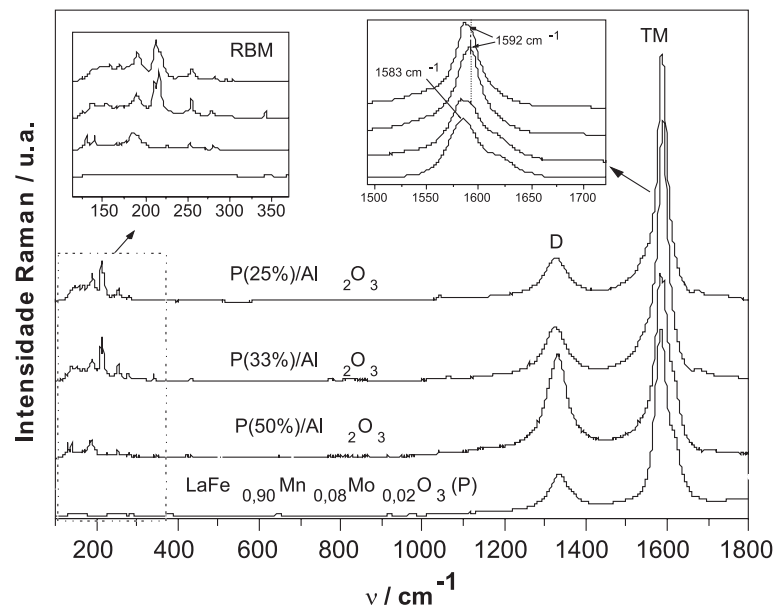

Figura 4. Espectros Raman (633 nm) obtidos após $\mathrm{CVD}$ com $\mathrm{CH} / 900{ }^{\circ} \mathrm{C}$ da perovskita LaFe ${ }_{0,90} \mathrm{Mn}_{0,08} \mathrm{Mo}_{0,02} \mathrm{O}_{3}$ antes e após dispersão de 50, 33 e 25\% em $\mathrm{Al}_{2} \mathrm{O}_{3}$

A Figura 5 mostra o acompanhamento da formação de $\mathrm{H}_{2}$ durante o processo CVD para os catalisadores perovskitas $\mathrm{LaFe}_{0.90} \mathrm{Mn}_{0.10} \mathrm{O}_{3}$, $\mathrm{LaFe}_{0,90} \mathrm{Mn}_{0,08} \mathrm{Mo}_{0,02} \mathrm{O}_{3} \mathrm{LaFe}_{0,90} \mathrm{Mn}_{0,08} \mathrm{Mo}_{0,02} \mathrm{O}_{3} 25$ e $50 \%$ em $\mathrm{Al}_{2} \mathrm{O}_{3}$ e o catalisador clássico $\mathrm{Fe}-\mathrm{Mo} / \mathrm{Al}_{2} \mathrm{O}_{3}$.

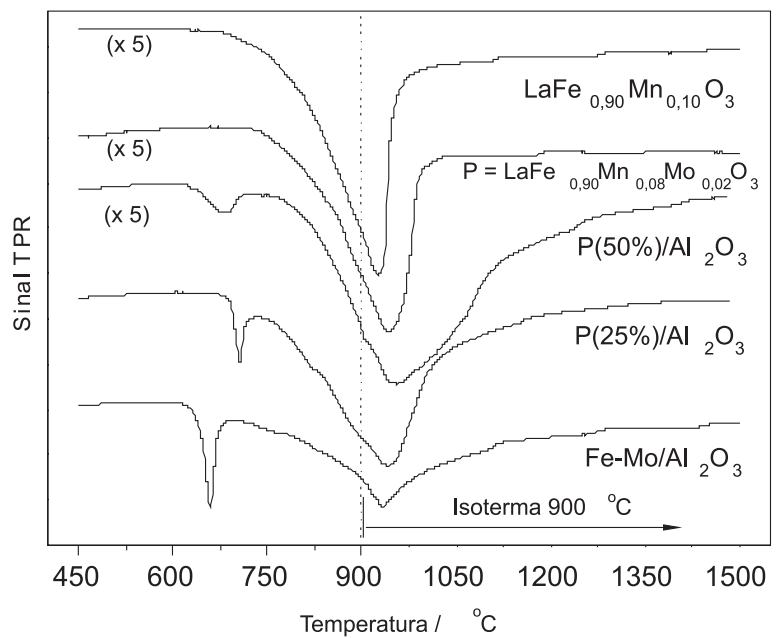

Figura 5. Acompanhamento da formação de $\mathrm{H}_{2}$ no processo CVD $\left(3^{a}\right.$ etapa) para os catalisadores $\mathrm{LaFe}_{0.90} \mathrm{Mn}_{0,10} \mathrm{O}_{3}, \mathrm{LaFe}_{0,90} \mathrm{Mn}_{0,08} \mathrm{Mo}_{0,02} \mathrm{O}_{3}$, $\mathrm{LaFe}_{0,90} \mathrm{Mn}_{0,08} \mathrm{Mo}_{0,02} \mathrm{O}_{3} 25$ e $50 \%$ em $\mathrm{Al}_{2} \mathrm{O}_{3}$ e catalisador clássico $\mathrm{Fe}-\mathrm{Mo}_{\mathrm{Al}} \mathrm{Al}_{2} \mathrm{O}_{3}$ (pré-redução com $\mathrm{H}_{2}(5 \%) / \mathrm{N}_{2}$ até $900{ }^{\circ} \mathrm{C}$ por $1 \mathrm{~h}$ )

A análise da Figura 5 mostra uma pequena produção de $\mathrm{H}_{2}$ que ocorre a 705 e $680{ }^{\circ} \mathrm{C}$ para as perovskitas suportadas $\mathrm{P}(25 \%) / \mathrm{Al}_{2} \mathrm{O}_{3}$ e $\mathrm{P}(50 \%) / \mathrm{Al}_{2} \mathrm{O}_{3}$, respectivamente. Este pico de formação de $\mathrm{H}_{2}$ a temperaturas relativamente baixas é similar ao observado a $660^{\circ} \mathrm{C}$ para o catalisador clássico $\mathrm{Fe}-\mathrm{Mo} / \mathrm{Al}_{2} \mathrm{O}_{3}$. É interessante observar a ausência deste pico de formação de $\mathrm{H}_{2}$ para o catalisador perovskita não suportado. Estes resultados reforçam a idéia de que este pico de 
$\mathrm{H}_{2}$ a baixa temperatura está relacionado com a formação de carbono amorfo, devido a uma fase de $\mathrm{Fe}$ altamente dispersa. As perovskitas puras não suportadas, $\mathrm{LaFe}_{0,90} \mathrm{Mn}_{0,10} \mathrm{O}_{3}, \mathrm{LaFe}_{0,90} \mathrm{Mn}_{0,08} \mathrm{Mo}_{0,02} \mathrm{O}_{3}$, tendem a formar partículas de Fe de maior tamanho e por isso não apresentam este pico de $\mathrm{H}_{2}$ a menores temperaturas.

Uma informação relevante que pode ser obtida a partir da Figura 6 é a quantidade de $\mathrm{H}_{2}$ formado durante a reação CVD. Esta quantidade está diretamente relacionada à quantidade de carbono formado no processo. A Figura 6 apresenta os rendimentos em carbono obtidos através de diferentes medidas, i.e., por ganho de massa após CVD, por perda de massa durante análise TG (em ar) e pela medida de $\mathrm{H}_{2}$ formado durante o experimento no sistema RTP. Os rendimentos indicam a quantidade de $\mathrm{C}$ formada em relação à quantidade de $\mathrm{Fe}$ no catalisador.

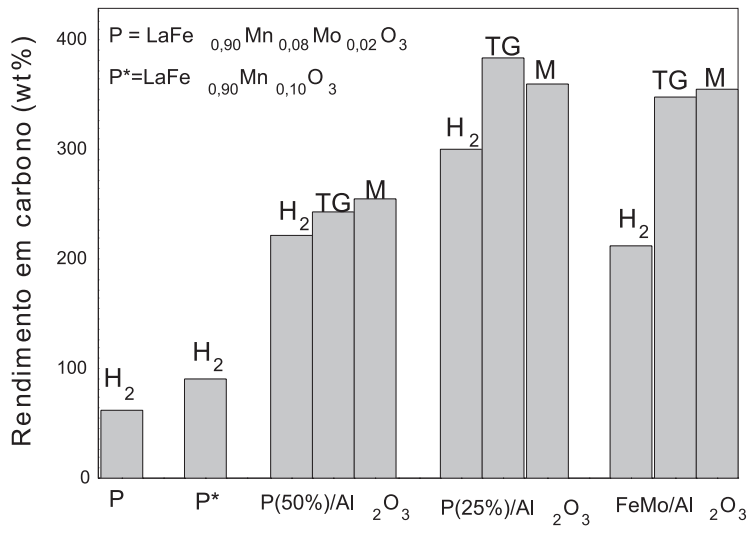

Figura 6. Relação entre as áreas das curvas de produção de $\mathrm{H}_{2}$ normalizada para os diferentes catalisadores testados

A análise dos dados obtidos pela medida de $\mathrm{H}_{2}$ mostra que os catalisadores perovskitas não suportadas produzem pouco carbono no processo. Por outro lado, maiores rendimentos de carbono são produzidos pelos catalisadores suportados, especialmente pelo $\mathrm{P}(25 \%) /$ $\mathrm{Al}_{2} \mathrm{O}_{3}$ que chegou a aproximadamente $300 \%$. A Figura 6 mostra também que os rendimentos obtidos através de medidas de massa e por TG estão muito próximos aos valores obtidos por medidas de $\mathrm{H}_{2}$.

De acordo com os dados MEV e Raman, os catalisadores que produziram NTC, especialmente os de parede simples, foram os que mostraram maiores rendimentos.

Outro aspecto importante a ser analisado é a inclinação do sinal tanto para a formação de $\mathrm{H}_{2}$ quanto para a fase de desativação Esta inclinação está diretamente relacionada à velocidade de formação de $\mathrm{H}_{2} \mathrm{e}$, portanto, à velocidade de formação dos depósitos de carbono ou de desativação do catalisador. ${ }^{23}$

Para este estudo cinético, considerou-se apenas o sinal de formação de $\mathrm{H}_{2}$ na fase isotérmica (Figura 5). O detalhe na Figura 7 mostra como as constantes $\mathrm{k}$ de formação de $\mathrm{H}_{2}$ e de desativação foram obtidas. Observa-se um comportamento aproximadamente linear, tanto para a fase de formação de $\mathrm{H}_{2}$ quanto para a fase de desativação do catalisador. Este comportamento linear sugere que tanto a formação de $\mathrm{H}_{2}$ quanto a desativação mostram uma velocidade constante. A partir destes resultados pode-se inferir que a formação de carbono na fase isotérmica a $900^{\circ} \mathrm{C}$, é processo com cinética de ordem zero. Isso provavelmente está relacionado à concentração constante de $\mathrm{CH}_{4}$ na corrente gasosa e à concentração da fase ativa de catalisador, que também é relativamente constante. Outro aspecto que chama a atenção é que a inclinação do sinal RTP na fase isotérmica muda repentinamente. Este comportamento sugere que o processo de desativação se inicia repentinamente, afetando a formação de carbono em todas as partículas de Fe presentes no sistema.
A comparação das inclinações dos sinais do detector do sistema RTP para os diferentes catalisadores testados, $\mathrm{LaFe}_{0.90} \mathrm{Mn}_{0.10} \mathrm{O}_{3}$, $\mathrm{LaFe}_{0,90} \mathrm{Mn}_{0,08} \mathrm{Mo}_{0,02} \mathrm{O}_{3}, \mathrm{LaFe}_{0,90} \mathrm{Mn}_{0,08} \mathrm{Mo}_{0,02} \mathrm{O}_{3} 25$ e $50 \%$ em $\mathrm{Al}_{2} \mathrm{O}_{3}$ e catalisador clássico Fe-Mo/Al ${ }_{2} \mathrm{O}_{3}$, é mostrada na Figura 7 .

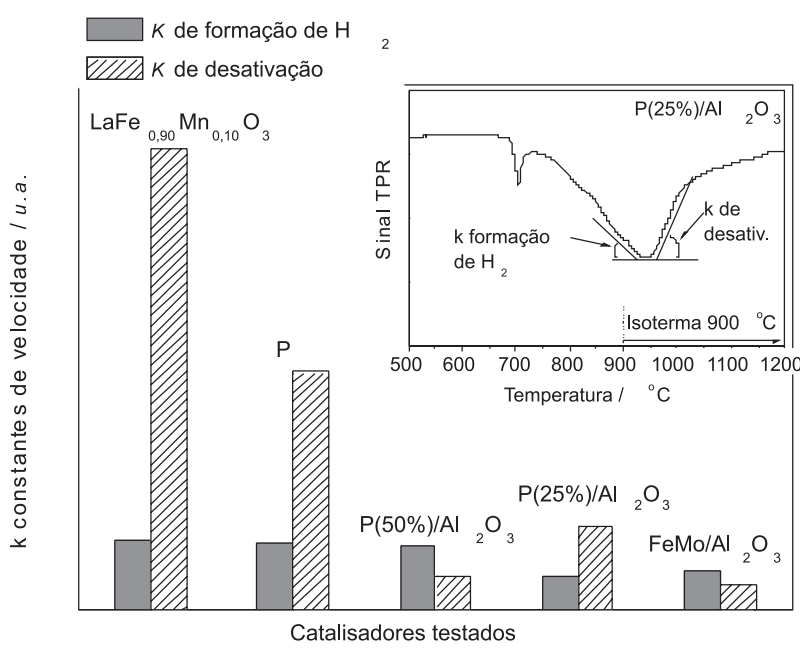

Figura 7. Relação entre as constantes de velocidade de formação de $\mathrm{H}_{2}$ e de desativação do catalisador para as diferentes amostras testadas

Os menores $\mathrm{k}$ de formação de $\mathrm{H}_{2}$ foram observados para os catalisadores que produzem NTPS, como $\mathrm{P}(25 \%) / \mathrm{Al}_{2} \mathrm{O}_{3}, \mathrm{P}(50 \%) / \mathrm{Al}_{2} \mathrm{O}_{3}$ e $\mathrm{Fe}-\mathrm{Mo} / \mathrm{Al}_{2} \mathrm{O}_{3}$. Estes mesmos catalisadores apresentam também as menores constantes $\mathrm{k}$ de desativação.

Por outro lado, para os catalisadores nos quais a velocidade de formação de $\mathrm{H}_{2}$ é alta, como para os catalisadores $\mathrm{LaFe}_{0,90} \mathrm{Mn}_{0,10} \mathrm{O}_{3} \mathrm{e}$ $\mathrm{LaFe}_{0,90} \mathrm{Mn}_{0,08} \mathrm{Mo}_{0,02} \mathrm{O}_{3}$, pouco carbono é formado, pois eles se desativam muito rápido. Estes dados sugerem que em catalisadores à base de ferro formas mais organizadas de carbono, tais como nanotubos, são formadas por processos mais lentos, enquanto carbono amorfo é formado por processos mais rápidos.

\section{CONCLUSÕES}

Os resultados preliminares aqui apresentados representam uma importante contribuição para o desenvolvimento da ciência dos nanotubos de carbono, pois abrem as portas para um novo método de estudo da cinética de formação dos NTC no processo CVD, através do uso do sistema de redução à temperatura programada (RTP). Esta nova técnica proporciona inúmeras possibilidades para o estudo da formação de NTC, tais como acompanhamento cinético da reação, estudo da desativação do catalisador, determinação do rendimento, entre outros.

\section{MATERIAL SUPLEMENTAR}

Encontram-se disponibilizadas em http://quimicanova.sbq.org.br, na forma de arquivo.PDF, com acesso livre, as microscopias eletrônicas de varredura e as curvas TG dos materiais obtidos após o processo CVD.

\section{AGRADECIMENTOS}

À FAPEMIG, PRPq/UFMG, ao CNPq, á CAPES pelo apoio financeiro; ao Prof. R. Martel da Universidade de Montreal pelos trabalhos preliminares executados em seu laboratório.

\section{REFERÊNCIAS}

1. Dupuis, A. C.; Prog. Mat. Sci. 2005, 50, 929. 
2. Terrones, M.; Int. Mater. Rev. 2004, 49, 325.

3. Veziri, C. M.; Pilatos, G.; Karanikolos, G. N.; Labropoulos, A.; Kordatos, K.; Kasselouri-Rigopoulou, V.; Kanellopouios, N. K.; Microp. Mesop. Mat. 2008, 110, 41.

4. Kouravelou, K. B.; Sotirchos, S. V.; Verykios, X. E.; Surf. Coat. Technol. 2007, 201, 9226.

5. Asai, K.; Nagayasu, Y.; Takane, K.; Iwamoto, S.; Yagasaki, E.; Ishii, K.; Inoue, M.; J. Jpn. Petrol. Inst. 2008, 51, 42.

6. Maeda, M.; Kamimura, T.; Matsumoto, K.; Appl. Phys. Lett. 2007, 90 , 043119.

7. Svrcek, V.; Kleps, I.; Cracioniou, F.; Paillaud, J. L.; Dintzer, T.; Louis, B.; Begin, D.; Pham-Huu, C.; Ledoux, M. J.; Le Normand, F.; J. Chem. Phys. 2006, 124, 184705.

8. Vera-Agullo, J.; Varela-Rizo, H.; Font, R.; Conesa, J. A.; Martin-Gullon, I.; J. Anal. Appl. Pyrolysis 2007, 79, 484.

9. Kim, D. H.; Jang, H. S.; Kim, C. D.; Cho, D. S.; Yang, H. S.; Kang, H. D.; Min, B. K.; Lee, H. R.; Nano Lett. 2003, 3, 863.

10. Kim, D. H.; Jang, H. S.; Kim, C. D.; Cho, D. S.; Kang, H. D.; Jee, J. G.; Lee, H. R.; Carbon 2003, 41, 583.

11. Kim, D. H.; Jang, H. S.; Kim, C. D.; Cho, D. S.; Jee, J. G.; Lee, H. R.; Nanotechnology 2003, 14, 46.

12. Nele, M.; Moreno, E. L.; Andrade, H. M. C.; Quim. Nova 2006, 29, 641
13. Kanervo, J.; PhD Thesis, Helsinki University of Technology, Finland, 2003.

14. Besselmann, S.; Freitag, C.; Hinrichsen, O.; Muhler, M.; Physical Chemistry Chem. Phys. 2001, 3, 4633.

15. Moura, F. C. C.; Tristão, J. C.; Lago, R. M.; Martel, R.; Catal. Today 2008, 133, 846

16. Tristão, J. C.; Dissertação de Mestrado, Universidade Federal de Minas Gerais, Brasil, 2007.

17. Tristão, J. C.; Pereira, M. C.; Moura, F. C. C.; Fabris, J. D.; Lago, R. M.; Mat. Res. 2008, 11, 233.

18. Cassel, A. M.; Raymakers, J. A.; Kong, J.; Dai, H. J.; J. Phys. Chem. B 1999, 103, 6484.

19. Zheng, M.; Su, B.; Liu, J.; Chem. Phys. Lett. 2000, 322, 321.

20. Delzeit, L.; Chen, B.; Cassel, A.; Stevens, R.; Nguyen, C.; Meyyappan, M.; Chem. Phys. Lett. 2001, 348, 368.

21. Oliveira, L. C. A.; Fabris, J. D.; Rios, R. R. V. A.; Mussel, W. N.; Lago, R. M.; Appl. Catal. A 2004, 259, 253.

22. Alvarez, L.; Righi, A.; Guillard, T.; Rols, S.; Anglaret, E.; Laplaze, D.; Sauvajol, J. L.; Chem. Phys. Lett. 2000, 316, 186.

23. Konieczny, A.; Mondal, K.; Wiltowski, T.; Dydo, P.; Int. J. Hydrogen Energy 2008, 33, 264. 


\section{SISTEMA RTP: UMA TÉCNICA PODEROSA PARA O MONITORAMENTO DA FORMAÇÃO DE NANOTUBOS DE CARBONO DURANTE O PROCESSO POR DEPOSIÇÃO DE VAPOR QUÍMICO}

Juliana Cristina Tristão, Flávia Cristina Camilo Moura e Rochel Montero Lago*

Departamento de Química, Instituto de Ciências Exatas, Universidade Federal de Minas Gerais, 31270-901 Belo Horizonte - MG, Brasil Karim Sapag

Laboratorio de Ciencias de Superficies y Medios Porosos, Universidade Nacional de San Luis, 5700, San Luis, Argentina
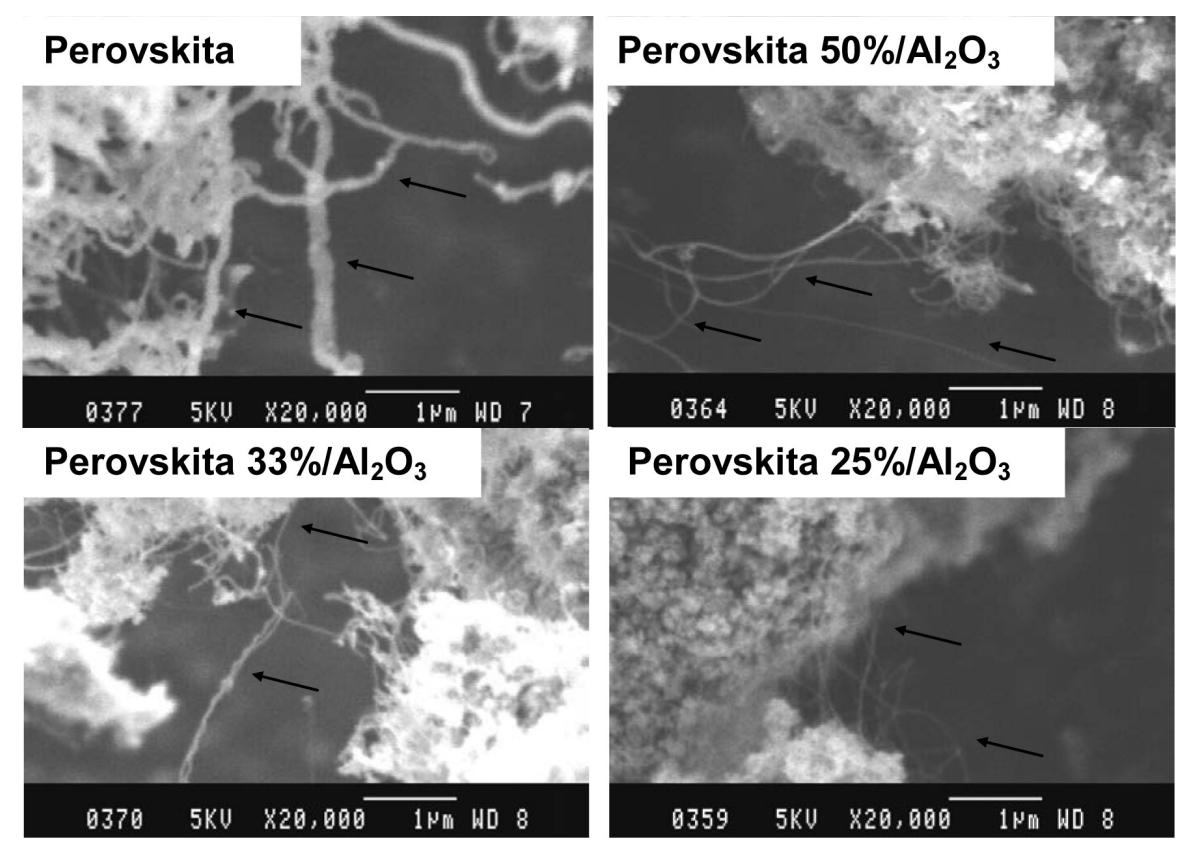

Figura 1S. Imagens MEV após CVD com CH $4900^{\circ} \mathrm{C}$ da perovskita $\mathrm{LaFe}_{0,90} \mathrm{Mn}_{0,08} \mathrm{Mo}_{0,02} \mathrm{O}_{3}$ antes e após dispersão em $\mathrm{Al}_{2} \mathrm{O}_{3}$ nas proporções de 50,33 e $25 \%$ em massa de perovskita

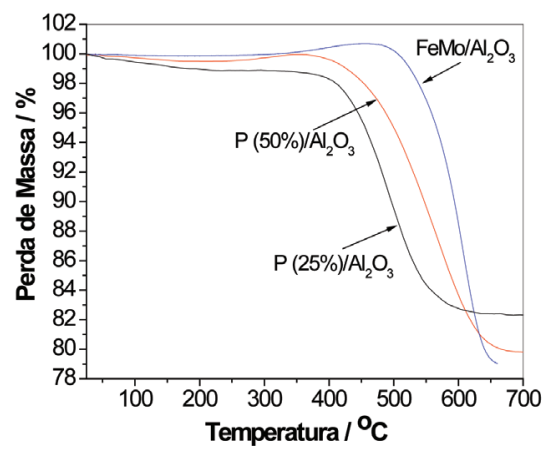

Figura 2S. Curvas TG para FeMo/Al $\mathrm{O}_{3}, \mathrm{P}(25 \%) / \mathrm{Al}_{2} \mathrm{O}_{3}$ e $\mathrm{P}(50 \%) / \mathrm{Al}_{2} \mathrm{O}_{3}$, após $\mathrm{CVD}$ com $\mathrm{CH}_{4} / 900{ }^{\circ} \mathrm{C}$, em ar 